\title{
Hydroxycamptothecin-loaded nanoparticles enhance target drug delivery and anticancer effect
} Anxun Wang*1 and $\mathrm{Su} \mathrm{Li}^{2}$

Address: ${ }^{1}$ Department of Oral and Maxillofacial Surgery, First Affiliated Hospital, Sun Yat-sen University, Guangzhou, China and ${ }^{2}$ Department of Medicine, Tumor Hospital, Sun Yat-sen University, Guangzhou, China

Email: Anxun Wang* - anxunwang@yahoo.com; Su Li - lisusu@tom.com

* Corresponding author

Published: 4 May 2008

BMC Biotechnology 2008, 8:46 doi:10.1/86/1472-6750-8-46
Received: 21 November 2007

Accepted: 4 May 2008

This article is available from: http://www.biomedcentral.com/1472-6750/8/46

(c) 2008 Wang and Li; licensee BioMed Central Ltd.

This is an Open Access article distributed under the terms of the Creative Commons Attribution License (http://creativecommons.org/licenses/by/2.0), which permits unrestricted use, distribution, and reproduction in any medium, provided the original work is properly cited.

\begin{abstract}
Background: Hydroxycamptothecin (HCPT) has been shown to have activity against a broad spectrum of cancers. In order to enhance its tissue-specific delivery and anticancer activity, we prepared HCPT-loaded nanoparticles made from poly(ethylene glycol)-poly( $\gamma$-benzyl-L-glutamate) (PEG-PBLG), and then studied their release characteristics, pharmacokinetic characteristics, and anticancer effects. PEG-PBLG nanoparticles incorporating HCPT were prepared by a dialysis method. Scanning electron microscopy (SEM) was used to observe the shape and diameter of the nanoparticles. The HCPT release characteristics in vitro were evaluated by ultraviolet spectrophotometry. A high-performance liquid chromatography (HPLC) detection method for determining HCPT in rabbit plasma was established. The pharmacokinetic parameters of HCPT/ PEG-PBLG nanoparticles were compared with those of HCPT.

Results: The HCPT-loaded nanoparticles had a core-shell spherical structure, with a core diameter of $200 \mathrm{~nm}$ and a shell thickness of $30 \mathrm{~nm}$. Drug-loading capacity and drug encapsulation were 7.5 and $56.8 \%$, respectively. The HCPT release profile was biphasic, with an initial abrupt release, followed by sustained release. The terminal elimination half-lives (t I/2 $\beta$ ) of HCPT and HCPT-loaded nanoparticles were 4.5 and $10.1 \mathrm{~h}$, respectively. Peak concentrations (Cmax) of HCPT and HCPT-loaded nanoparticles were 2627.8 and $1513.5 \mu \mathrm{g} / \mathrm{L}$, respectively. The apparent volumes of distribution of the HCPT and HCPT-loaded nanoparticles were 7.3 and $20.0 \mathrm{~L}$, respectively. Compared with a blank control group, Lovo cell xenografts or Tca8II3 cell xenografts in HCPT or HCPT-loaded nanoparticle treated groups grew more slowly and the tumor doubling times were increased. The tumor inhibition effect in the HCPT-loaded nanospheretreated group was significantly higher than that of the HCPT-treated group $(p<0.01)$. Tumor inhibition in the control group by PEG-PBLG nanoparticles was not observed $(p>0.05)$.

Conclusion: Compared to the HCPT- and control-treated groups, the HCPT-loaded nanoparticle-treated group showed a more sustained release, a longer circulation time, increased delivery to tissue, and an enhanced anticancer effect. HCPT-loaded nanoparticles appear to change the pharmacokinetic behavior of HCPT in vivo.
\end{abstract}




\section{Background}

In recent years, microspheres, liposomes, and biodegradable polymers have been used in site-specific drug delivery systems [1-3]. Hydrophilic-hydrophobic diblock copolymers exhibit amphiphilic behavior and form micelles with core-shell architecture. The hydrophobic block forms the inner core, which acts as a drug incorporation site, especially for the hydrophobic drugs. The hydrophilic block forms the hydrated outer shell, which plays a role in preventing uptake by the reticuloendothelial system (RES) $[2,4]$. The predominant characteristics of these copolymers that have been reported include solubilization of hydrophobic drugs, sustained release, selective targeting, and lower interactions with the RES $[2,4,5]$. Nanoparticles made from poly $(\gamma$-benzyl L-glutamate) (PBLG) and poly(ethylene oxide) (PEG) are hydrophilichydrophobic diblock copolymers which have these predominant characteristics [6,7]. Thus, this PEG-PBLG copolymeric carrier may serve as an appropriate vehicle for drug delivery $[6,7]$.

The anticancer activity of camptothecin (CPT) and its natural and synthetic analogs has been shown in a broad spectrum of cancers, including leukemias and cancers of the liver, stomach, breast, and colon [8-10]. Among natural CPTs, 10-hydroxycamptothecin (HCPT) has been shown to be more active and less toxic $[8,9,11]$; however, natural HCPT is in a lactone form and is water-insoluble. One way to improve the solubility of HCPT is to change the lactone form to the carboxylate form by adding $\mathrm{NaOH}$. However, this leads to less activity and more unwanted toxicity $[8,9]$. At the same time, HCPT has a short half-life in vivo and poor biodistribution [12].

To improve the solubility of CPT analogs, the lactone form of the analogs was incorporated into liposomes or nanoparticles [13-15]. These delivery systems show favorable pharmacokinetics and biodistribution [13-15]. In the present study, we prepared HCPT-loaded PEG-PBLG nanoparticles and investigated the in vitro release, pharmacokinetics, and anticancer effect. Our results showed that HCPT-loaded nanoparticles changed the pharmacokinetic behavior of HCPT in vivo. The HCPT-loaded nanoparticles had a more sustained release, a longer circulation time, increased delivery to tissue, and an enhanced anticancer effect.

\section{Results}

\section{Characteristics of HCPT-loaded nanoparticles}

In this study, HCPT-loaded PBLG/PEG nanoparticles were prepared using dialysis. After analysis by UV spectrophotometey, we found that the solution of HCPT in a standard sample, or the simple mixture of a solution of HCPT and PBLG/PEG, had high absorbency in the wavelength range, $326-368 \mathrm{~nm}$ (Fig. 1A,B). However, the absorb- ency of the solution of HCPT-loaded PBLG/PEG nanoparticles was greatly decreased (Fig. 1C), representing the formation of HCPT-loaded PEG-PBLG nanoparticles. On the basis of absorbency at $326 \mathrm{~nm}$, the drug-loading capacity and drug encapsulation of HCPT-loaded nanoparticles were 7.5 and $56.8 \%$, respectively.

The morphology of HCPT-loaded nanoparticles (Fig. 1D) was found to be a core-shell structure that was spherical or elliptical, with a smooth surface. The hydrophobic central core, the grayish area inside the bright white ring, was approximately $200 \mathrm{~nm}$ in diameter. The hydrophilic shell, the bright white ring, was approximately $30 \mathrm{~nm}$ in thickness.

\section{Abrupt-sustained release of HCPT-loaded nanoparticles}

The standard curve of a HCPT solution was derived from the following equation: $\mathrm{y}=10.7 \mathrm{x}+0.0056(\mathrm{r}=0.9999)$. HCPT release from PEG-PBLG nanoparticles at pHs 6.86 and 9.18 in vitro is shown on Fig. 2. The HCPT release profile was biphasic, with an initial abrupt release, followed by sustained release. Abrupt release occurred at $2 \mathrm{~h}$, and $1 / 3$ of the loaded HCPT was released by that time. Then, the release of HCPT entered sustained release following initial abrupt release. After 96 h, 3/5 of the loaded HCPT was still enveloped in the nanoparticles. In this release study, HCPT-loaded nanoparticles showed a quicker release pattern in the more alkaline condition.

\section{Pharmacokinetic characteristics of HCPT-loaded nanoparticles}

The standard curve for HCPT in rabbit plasma was derived from the following equation: $\mathrm{y}=2.75 \mathrm{x}+2.90(\gamma>$ $0.9999)$, where $\mathrm{Y}$ is the concentration $(\mu \mathrm{g} / \mathrm{L})$ and $\mathrm{X}$ is the peak height. The limit of determination was $2 \mu \mathrm{g} / \mathrm{L}$. The mean plasma concentrations over time for the free HCPT and the HCPT-loaded nanoparticles are illustrated in Fig. 3 . The HCPT release profile from HCPT-loaded nanoparticles was biphasic with an initial abrupt release, followed by sustained release. Abrupt release occurred at $1 \mathrm{~h}$ and the peak concentration was $1513.5 \mu \mathrm{g} / \mathrm{L}$. During the sustained release, the plasma concentration was between 7.4 $-84.7 \mu \mathrm{g} / \mathrm{L}$. As shown in Table 1, the pharmacokinetics of HCPT changed after it was loaded into PEG-PBLG nanoparticles. The terminal elimination half-life was longer, the peak concentration decreased, and the apparent volume of distribution increased.

\section{Tumor inhibition effect of HCPT-loaded nanoparticles in vivo}

Tumor-associated swellings were visible in all the mice. As demonstrated by the tumor growth curve of Lovo (Fig. 4A) and Tca8113 cell xenografts (Fig. 4B), xenograft growth was fast in the blank and PEG-PBLG control groups, but significantly depressed in the HCPT- or 


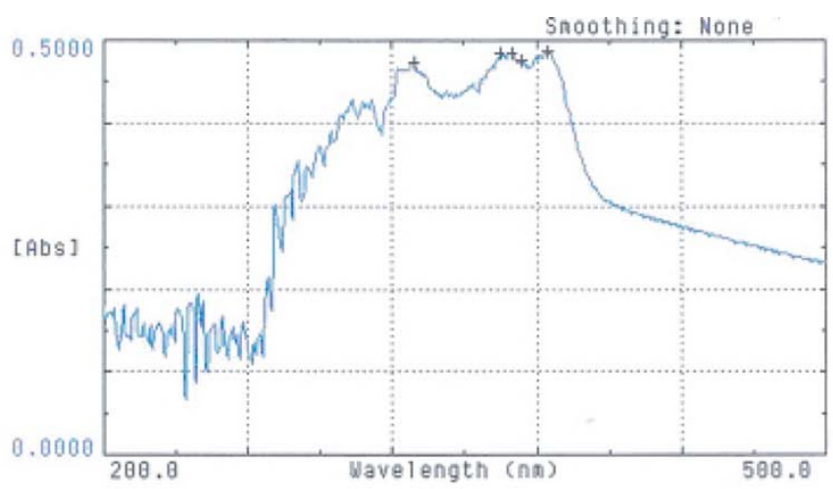

A

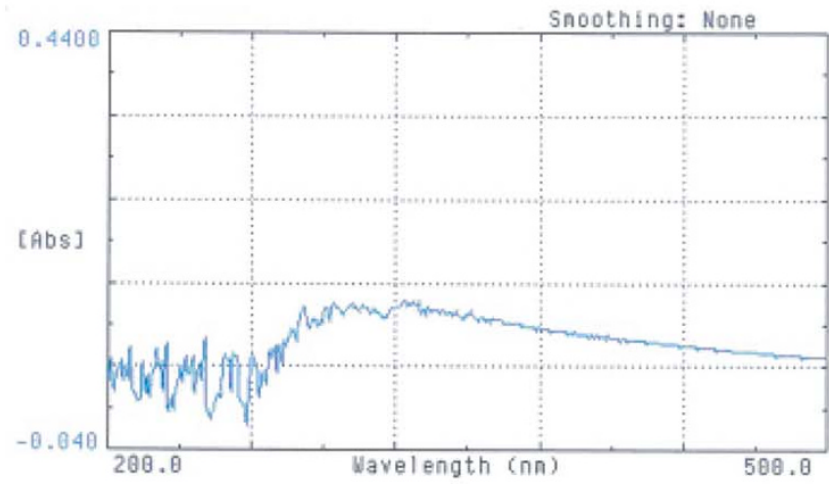

C

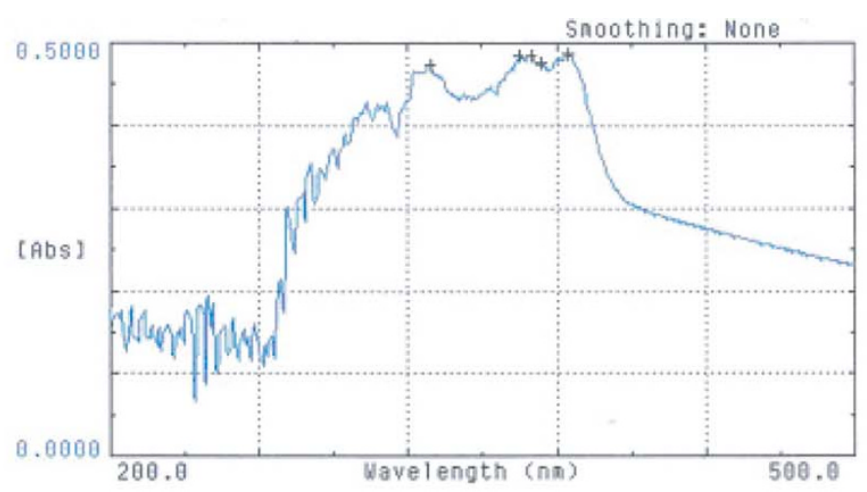

B

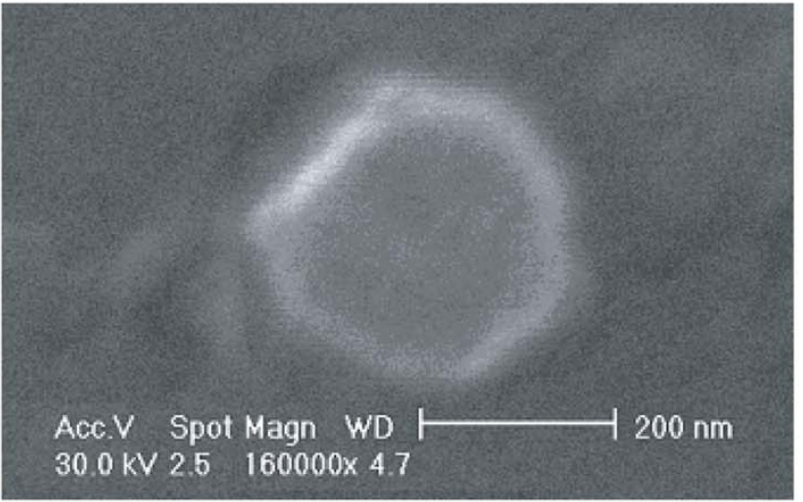

$\mathrm{D}$

\section{Figure I}

Characteristics of HCPT-loaded nanoparticles. (A-C): UV spectrum of HCPT detected by ultraviolet spectrophotometry. At wavelengths of 326 and $368 \mathrm{~nm}$, both the HCPT standard sample (A) and the mixture of HCPT and PBLG/PEG (B) had high absorbency, but the absorbency of HCPT-loaded PBLG/PEG nanoparticles (C) was greatly decreased. (D) The morphology of HCPT-loaded nanoparticles was found under scanning electron microscopy $(\times 160000)$ to be a core-shell structure, spherical or elliptical, with a smooth surface. The hydrophobic central core was a grayish area, approximately $200 \mathrm{~nm}$ in diameter. The hydrophilic shell was a bright white ring, approximately $30 \mathrm{~nm}$ thick.

HCPT/PEG-PBLG-treated groups. As shown in Table 2, tumor doubling time lengthened with treatment. The inhibition rate for HCPT alone was between 60 and 70\%, but was greater than $80 \%$ for the HCPT-loaded nanoparticles. The tumor volumes of the HCPT and HCPT-loaded nanoparticle-treated groups were significantly less than those of the blank and PEG-PBLG control groups $(\mathrm{P}<$ $0.01)$. There was also significantly more tumor inhibition in the HCPT-loaded nanoparticle treated group than in the HCPT treated group $(\mathrm{P}<0.01)$. However, there was no significant difference in tumor inhibition between the two control groups $(\mathrm{P}>0.05)$. No significant toxicity was observed in any of the groups.

\section{Discussion}

Drug-loaded nanoparticles made from natural and synthetic macromolecular materials that are biocompatible and biodegradable have been used for controlling the release of drugs and changing pharmacokinetics and the targets of drug action [1-3]. Nanoparticles have been used

Table I: The pharmacokinetic parameters of HCPT or HCPT-loaded nanoparticles after IV administration at a single dose of I 2 mg/kg in rabbit

\begin{tabular}{lccccc}
\hline & $\mathrm{t}_{1 / 2}(\mathrm{~h})$ & $\mathrm{C}_{\max }(\mu \mathrm{g} / \mathrm{L})$ & $\mathrm{T}_{\max }(\mathrm{h})$ & $\mathrm{V}_{\mathrm{d}}(\mathrm{L})$ & $\mathrm{AUC}(\mu \mathrm{g} \cdot \mathrm{h} / \mathrm{L})$ \\
\hline HCPT & 4.5 & 2627.8 & 0 & 7.3 & 2459.0 \\
HCPT/PEG-PBLG & 10.1 & 1513.5 & $\mathrm{I}$ & 20 & 2175.9 \\
\hline
\end{tabular}

$T_{1 / 2 \beta}$ : terminal elimination half-life; $C_{\max }$ : peak concentration; $T_{\max }:$ peak time; AUC: area under the curve ; $V_{\mathrm{d}}$ : apparent volume of distribution. 


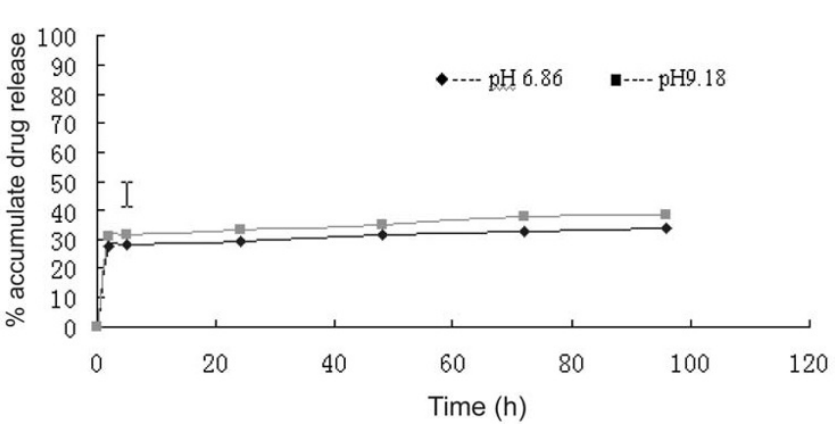

Figure 2

Release characteristics of HCPT from PEG-PBLG nanoparticles in vitro in different buffers. The HCPT release profile was biphasic with an initial abrupt release, followed by sustained release. The abrupt release occurred at 2 $h$ and $I / 3$ of the loaded-HCPT was released during this period. Then, the HCPT entered the sustained release; after $96 \mathrm{~h}, 3 / 5$ of the loaded-HCPT was still enwrapped in the nanoparticles. In this release study, HCPT-loaded nanoparticles also showed a quick release pattern in the alkaline condition.

to load anticancer drugs to enhance their anticancer effect and decrease their toxicity $[14,15]$, especially for waterinsoluble drugs. Nanoparticles made from PEG and PBLG, which are biocompatible and biodegradable macromolecule materials, have been used to load drugs [6,7]. In this study, HCPT-loaded PEG-PBLG nanoparticles were prepared by dialysis, and the drug loading capacity was $7.5 \%$, which was obviously higher than that of HCPTloaded polybutylcyanoacrylate nanoparticles (1.22\%) prepared by the adsorption-enwrapping method [16].

Like many other copolymer nanoparticles [6,7], the shape of HCPT-loaded nanoparticles was mostly spherical or ellipsoid. Close observation of SEM photographs revealed grayish and bright white profiles in the copolymer nanoparticles, which indicated that they are of the core shell type. The grayish center portion, which was $200 \mathrm{~nm}$ in diameter, was assigned to the core of the hydrophobic PBLG, and the bright white ring, which was $30 \mathrm{~nm}$ in thickness, was assigned to the shell of the hydrophilic
PEG. Studies have revealed that nanoparticles are not easily phagocytized by phagocytes when the thickness of the PEG layer is $10 \mathrm{~nm}$ for every $100 \mathrm{~nm}$ thickness of micelles [17]. Thus, the size of the nanoparticles was suitable to avoid uptake by the RES.

Numerous data have shown that the release of drugs from nanoparticles is biphasic, with abrupt and sustained release components [18]. Abrupt release includes release of the drug adsorbed at the surface of the nanoparticles or diffused from the polymer matrix. Abrupt release enables the drug to quickly reach effective blood concentrations. Sustained release is the release of the drug that was enwrapped inside the nanoparticles and occurs when the nanoparticles biodegrade, or diffuse from the polymer matrix. Sustained release is advantageous to maintain effective blood concentrations of the drug. In this study, the release profile of the HCPT-loaded nanoparticles also consisted of an abrupt release and a sustained release. The HCPT-loaded nanoparticles also showed a quick release pattern in the alkaline condition in vitro. This may be related to the fact that the lactone form of HCPT converts to the carboxylate form in the alkaline condition and becomes water-soluble.

HCPT is an inhibitor of topoisomerase I (Topo I). Other researchers have revealed that only the lactone form of HCPT can form a stable compound with Topo I and DNA, which may be responsible for its anticancer effect [8]. The carboxylate form of HCPT has a low anticancer effect, high toxicity, and poor stability. Previous pharmacokinetic studies with HCPT have indicated that HCPT has a short half-life, a poor affinity for tissue, and a higher combination rate with plasma protein $[12,19]$. In this study, we found the same results. Therefore, to improve the anticancer effect and decrease the toxicity of HCPT, many researchers have investigated a new preparation of HCPT $[13,15,16]$. Williams et al. [15] prepared SN-38- (an active compound of irinotecan) loaded phospholipid-PEG nanoparticles by the solvent-evaporation method, which enhanced the lactone ring stability in the presence of human serum albumin and prolonged the existence of the

Table 2: The anticancer effect of HCPT-loaded nanoparticles in the treatment of xenografts

\begin{tabular}{|c|c|c|c|c|c|c|}
\hline & \multicolumn{3}{|c|}{ Lovo cells xenograft } & \multicolumn{3}{|c|}{ Tca8I I 3 cells xenograft } \\
\hline & TDT (d) & IR (\%) & $\mathrm{TV}\left(\mathrm{cm}^{3}\right)$ & TDT (d) & IR (\%) & $\mathrm{TV}\left(\mathrm{cm}^{3}\right)$ \\
\hline Blank control & 3.0 & 0 & $4.336 \pm 0.485$ & 3.5 & 0 & $3.888 \pm 0.547$ \\
\hline PEG-PBLG & 2.9 & 0 & $4.206 \pm 0.308^{*}$ & 3.6 & 0 & $3.944 \pm 0.179 *$ \\
\hline HCPT & 4.3 & $70.0 \%$ & $1.299 \pm 0.082^{\#}$ & 4.5 & $59.8 \%$ & $1.564 \pm 0.286 \#$ \\
\hline HCPT/PEG-PBLG & 4.9 & $83.8 \%$ & $0.701 \pm 0.067 \# \S$ & 4.9 & $85.6 \%$ & $0.559 \pm 0.062 \# \S$ \\
\hline
\end{tabular}

TDT: tumor doubling time; IR: inhibition rate; TV: tumor volume at day $2 \mathrm{I}$ for Lovo cells xenograft or day 34 for Tca8I 3 cells xenograft

*: compared with blank: $P=0.45$ I or 0.765 (front:Lovo xenograft; the latter:Tca8 I I 3 xenograft); \#: compared with blank: $P=0.000$;

$\S$ : Compared with HCPT: $P=0.002$ or 0.000 (front:Lovo xenograft; the latter:Tca8 I I 3 xenograft) 


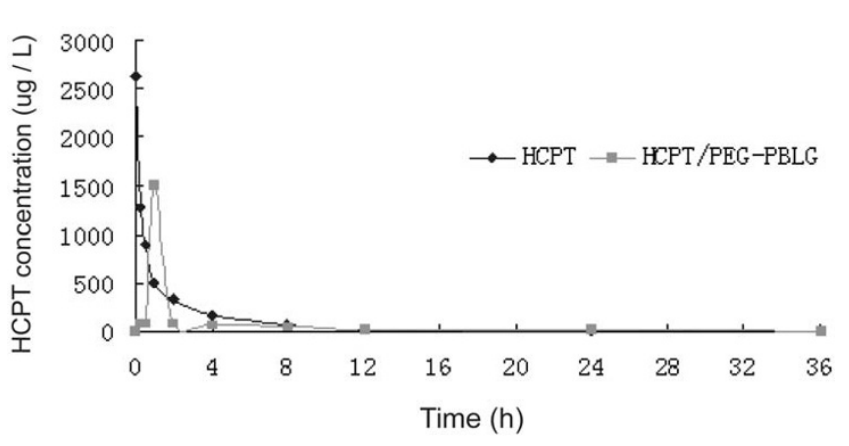

Figure 3

The mean plasma concentration of HCPT after IV administration of HCPT or HCPT/PEG-PBLG nanospheres at a single dose of $12 \mathrm{mg} / \mathrm{kg}$. The HCPT release profile from HCPT-loaded nanoparticles showed a biphasic with an initial abrupt release, followed by a sustained release. The abrupt release occurred at $\mathrm{I} h$ and the peak concentration was $15 \mathrm{I} 3.5 \mu \mathrm{g} / \mathrm{L}$. The release then became sustained, with a plasma concentration between 84.7 and $7.4 \mu \mathrm{g} / \mathrm{L}$.

active drug (lactone form) and the half-life in vivo. HCPTloaded nanoparticles prepared by Zhang [16] were targeted to the liver and had a sustained release effect. In our study, the pharmacokinetic parameters of HCPT changed after it was enveloped in the PEG-PBLG nanoparticles. Compared with HCPT, the pharmacokinetic parameters for the HCPT/PEG-PBLG nanoparticles had the following changes: 1) the terminal elimination half-life increased, 2) the peak concentration decreased, and 3) the apparent volume of distribution increased. These results indicate that the HCPT-loaded nanoparticles have the following characteristics: 1) sustained release, 2) prolonged half-life, and 3 ) increased affinity to tissue. Therefore, they have the properties of an ideal new preparation of HCPT. The studies of Li et al. [7] and Jeong et al. [6] have also shown that 5-fluorouracil- or adriamycin-loaded PEG-PBLG nanoparticles have similar pharmacokinetic characteristics.

HCPT has known clinical efficacy against a variety of solid tumors in humans $[8,9]$. In this study, free HCPT inhibited the xenograft growths of colon cancer and oral squamous cell carcinoma, and prolonged the tumor doubling times. These findings were also demonstrated in our previous studies $[9,20]$. Compared with free HCPT, HCPTloaded nanoparticles had a higher inhibitory effect on colon cancer and oral squamous cell carcinoma. Williams et al. [15] reported a similar result; after treatment with SN-38-loaded phospholipd-PEG, the anticancer effect against HT-29 colon xenografts was higher compared to CPT-11. The higher anticancer effect of HCPT-loaded nanoparticles may be due to one or more of the following reasons. First, the HCPT-loaded nanoparticles have sustained release, a prolonged half-life, and increase the apparent volume of distribution. These characteristics may increase the exposure time of the drug to tumor tissues. Second, the HCPT-loaded nanoparticles stabilize the lactone form of HCPT, which inhibits the activity of Topo I. The longer the period of stabilization, the stronger the anticancer effect. Third, tumor tissue has an abundant blood supply and tumor cells exhibit higher phagocytotic ability. Together, these characteristics would make nanoparticles more likely to enter the tumor cells and would improve the anticancer effect.

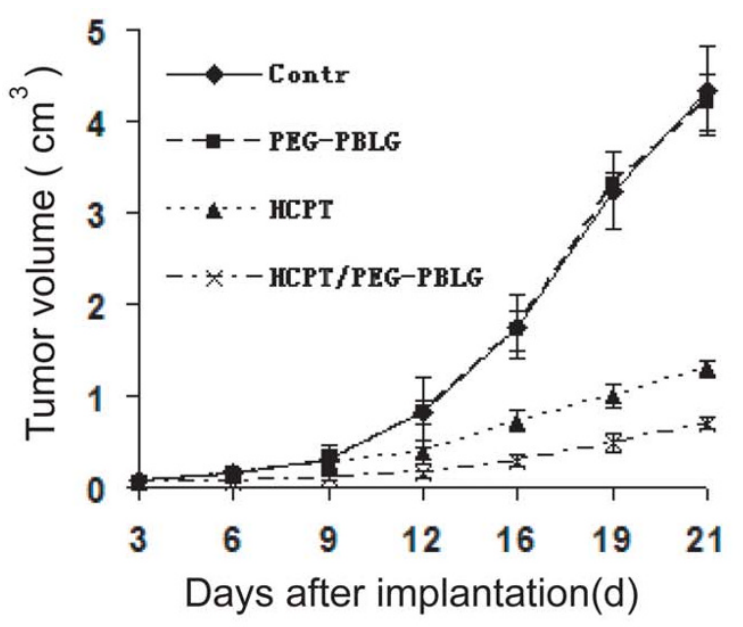

$4 \mathrm{~A}$

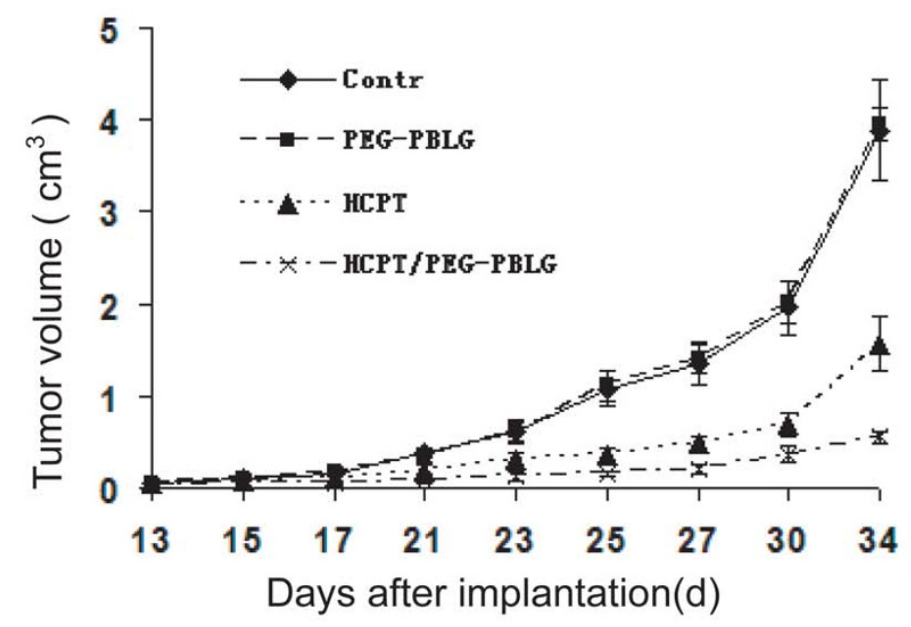

$4 \mathrm{~B}$

Figure 4

The tumor growth curve of Lovo cell xenografts (4A) or Tca8 I I 3 cell xenografts (4B). Xenografts grew quickly in the blank and PEG-PBLG control groups, but growth was significantly slowed in the HCPT- and HCPT/PEG-PBLG-treated groups. 


\section{Conclusion}

From this research, we found that PEG-PBLG nanoparticles are useful for the solubilization and sustained release of HCPT. HCPT-loaded PEG-PBLG nanoparticles improved the tissue-specific delivery and the anticancer effect of HCPT by changing the pharmacokinetic behavior of HCPT in vivo.

\section{Methods \\ Materials}

PEG-PBLG block copolymers were prepared by polymerization of $\gamma$-benzyl L-glutamate N-carboxyanhydride $(\gamma$ BLG NCA) initiated with mono amine-terminated PEG in a methylene dichloride solution by the method described previously [7]. HCPT (lactone form) powder (> 98.5\% purity) and HCPT liquid injection were obtained from Huangshi Lishizhen Pharmaceutical Co. (Hubei, China). All other reagents were of analytical grade. Human colon cancer cells (Lovo cell line) or oral squamous carcinoma cells (Tca8113 cell line) were grown in RPMI 1640 (GIBCO, USA) with 10\% fetal calf serum (GIBCO), 100 units/ml penicillin $\mathrm{G}$, and $100 \mu \mathrm{g} / \mathrm{ml}$ streptomycin at $37^{\circ} \mathrm{C}$ in $5 \% \mathrm{CO} 2$. New Zealand rabbits (2-3 kg) and SPF BALB/c nude mice, $6-8$ weeks of age (20-30 g) were purchased from the Animal Center of Sun Yat-sen University. Animal experiments were performed with the permission of the Animal Ethical Commission of Sun Yat-sen University.

\section{Preparation and identification of HCPT-loaded nanoparticles}

HCPT-loaded PEG-PBLG nanoparticles were prepared by dialysis, as described previously [7]. Briefly, PEG-PBLG diblock copolymer and HCPT (1:1 W/W) were dissolved in N,N-dimethylformamide (DMF), then dialyzed using a dialysis bag (molecular cut-off $3500 \mathrm{~g} / \mathrm{mol}$; Spectrum Medical Industries, Inc., Houston, TX) against double-distilled water for $24 \mathrm{~h}$. The solution inside the dialysis bag was centrifuged and supernatant (nanoparticles) was filtered through a $0.45 \mu \mathrm{m}$ filter. A 640 UV spectrophotometer (Beckman) was used to identify the HCPT-loaded PEG-PBLG nanoparticles at the wavelengths, 200-400 $\mathrm{nm}$. The morphology of nanoparticles was observed using a scanning electron microscope (SEM, HITACHI-600; Japan).

\section{Drug-loading capacity and drug encapsulation}

HCPT-loaded PEG-PBLG nanoparticles were added into the dialysis bag, which was placed in DMF. The solution outside the dialysis bag was stirred at $37^{\circ} \mathrm{C}$ for $3 \mathrm{~h}$ and then the drug concentration was measured using a UV spectrophotometer at $326 \mathrm{~nm}$. Absorbency of the solution (A) was used to calculate the drug-loading capacity and drug encapsulation according to the following formulae: drug loading capacity $=M_{\text {HСРТ }} / M_{\text {HCPT/PEG-PBLG }}$ and drug encapsulation $=M_{\mathrm{HCPT}} / M_{\text {drug devoted }}$, where $M_{\mathrm{HCPT}}$ was the drug content of the detected solution $\left(M_{\mathrm{HCPT}}=D_{\mathrm{HCPT}} \times V\right.$, $\left.D_{\text {HСРT }}=A_{\text {sample }} / A_{\text {standard }}\right) \times D_{\text {standard }} D$ : concentration, $V$ : volume), $M_{\text {HCPT/PEG-PBLG }}$ was the quantity of the detected solution of HCPT/PEG-PBLG nanoparticles, and $M_{\text {drug }}$ devoted was the initial quantity of HCPT.

\section{In vitro release}

HCPT-loaded nanoparticles were added to a dialysis bag and then introduced into a vial with PBS at different $\mathrm{pHs}$ (6.86 and 9.18). The medium was stirred at $94 \pm 4$ revolutions $/ \mathrm{min}$ at $37^{\circ} \mathrm{C}$. At the indicated time intervals (observed until $96 \mathrm{~h}$ ), the medium was removed and replaced with fresh PBS. The absorbency of samples of these replaced media was detected by an UV spectrophotometer at $326 \mathrm{~nm}$. The released HCPT in these replaced media at different time intervals was calculated from the standard curve, which was set up in the same way. Then, the release curve of HCPT-loaded nanoparticles was described.

\section{Pharmacokinetic study of HCPT-loaded nanoparticles in rabbit plasma}

Six New Zealand rabbits were randomized into two groups. After 12 hours of fasting, a bolus of the sample, equivalent to $12 \mathrm{mg} / \mathrm{kg}$ of HCPT or HCPT-loaded nanoparticles, was injected intravenously into each rabbit. Blood samples were withdrawn from the aural vein at the indicated time intervals. After centrifugation, the plasma supernatant was added to acetic acid $(\mathrm{pH} 3)$ to produce the lactone form of HCPT. Then, cold methanol-acetonitrile $(1: 1, \mathrm{v} / \mathrm{v})$ was used to precipitate proteins. After centrifuging at $10,000 \mathrm{r} / \mathrm{min}$ for $5 \mathrm{~min}$ at $4^{\circ} \mathrm{C}, 50 \mu \mathrm{L}$ of the supernatant were injected into the high-performance liquid chromatograph (HPLC, HP1100; Agilent) to determine the lactone concentration. The analytical column used was Hypersil C18 ( $5 \mu \mathrm{m}$, ID $4.6 \mathrm{~mm} \times 300 \mathrm{~mm}$ ). The mobile phase was $0.075 \mathrm{~mol} / \mathrm{L}$ ammonium acetate buffer (pH 6.4)/acetonitrile $(78: 22[\mathrm{v}: \mathrm{v}])$. The column was eluted at a flow rate of $1.0 \mathrm{~mL} / \mathrm{min}$ at room temperature; and the effluent was monitored spectrofluorometrically with an excitation wavelength of $269 \mathrm{~nm}$ and an emission wavelength of $550 \mathrm{~nm}$. The concentrations of HCPT were calculated based on the standard curve, which was set up using standard HCPT solution, and the pharmacokinetic parameters of HCPT distribution were estimated using 3 p87 programs, or calculated with open two-compartment models.

\section{Tumor inhibition effect of HCPT-loaded nanoparticles in vivo}

The xenograft model of colon cancer was established subcutaneously in the right flank of BALB/c nude mice. After xenografts about $5 \mathrm{~mm}$ in diameter formed (third day), the mice were randomly assigned to 4 groups $(n=8)$ as 
follows: control, PEG-PBLG, HCPT, and HCPT/PEGPBLG. Then HCPT or HCPT/PEG-PBLG, $3 \mathrm{mg} / \mathrm{kg}$, was intraperitoneally injected daily for 7 times. In the control group, the same volume of PBS or PEG-PBLG was injected intraperitoneally. Mice were sacrificed on day 21. Tumor size was measured by calipers (length and width) every 3 days. The tumor volume $\left(V=1 / 2\right.$ length $\times$ width $\left.^{2}\right)$ was calculated and the tumor growth curve was generated $(\mathrm{y}=\mathrm{A}$ $\left.\mathrm{e}^{\mathrm{k} t}\right)$. The tumor doubling time $(T=\ln 2 / K, \mathrm{k}$ : growth rate) and inhibition rate on day 21 were calculated. The inhibition rate was calculated as follows: (1-the volume change of experiment group/the volume change of control group) $\times 100 \%$. The establishment, grouping, and treatment protocol of the Tca8113 cell xenograft (oral squamous cell carcinoma) was similar to the Lovo cell xenograft, except for the following differences: 1) treatment began on day 8 , 2 ) the drug was injected every 2 days for 8 times (16 days), and 3 ) the mice were sacrificed on day 34 . The anti-tumor activity was evaluated as described above.

\section{Statistical analysis}

In all cases, experiments were replicated in triplicate and data represent mean \pm s.d. (standard deviation). Statistical analysis of the inhibitory effect on tumor growth was performed using one-way analysis of variance. $P<0.05$ denoted significance in all cases.

\section{List of abbreviations used}

HCPT: hydroxycamptothecin; PBLG: $\operatorname{poly}(\gamma$-benzyl 1glutamate); PEG: poly(ethylene glycol); SEM: scanning electron microscope; HPLC: high-performance liquid chromatography; RES: reticuloendothelial system; DMF: $\mathrm{N}, \mathrm{N}$-dimethylformamide; $\mathrm{t}_{1 / 2}$ : elimination half-life; $\mathrm{C}_{\max }$ : peak concentration; $\mathrm{T}_{\max }$ : peak time; AUC: area under the concentration-time curve; $\mathrm{V}_{\mathrm{d}}$ : distribution volume; TDT: tumor doubling time; IR: inhibition rate; TV: tumor volume at day 21 for Lovo cell xenografts or at day 34 for Tca 8113 cell xenografts.

\section{Competing interests}

The authors declare that they have no competing interests.

\section{Authors' contributions}

AW and SL were responsible for experimental design and completion of all laboratory work presented in this article. The manuscript was drafted by AW. All authors approved and read the final manuscript.

\section{Acknowledgements}

This work was supported by research grants from the Natural Science Foundation of Guangdong Province, No.021865, and the Science and Technique Project of Guangdong Province, No. 2004B30901002.

\section{References}

I. Mainardes RM, Silva LP: Drug delivery systems: past, present, and future. Curr Drug Targets 2004, 5(5):449-455.
2. Zentner GM, Rathi R, Shih C, McRea JC, Seo MH, Oh H, Rhee BG, Mestecky J, Moldoveanu Z, Morgan M, Weitman S: Biodegradable block copolymers for delivery of proteins and water-insoluble drugs. J Control Release 200I, 72(I-3):203-2I5.

3. Wang MD, Shin DM, Simons JW, Nie S: Nanotechnology for targeted cancer therapy. Expert Rev Anticancer Ther 2007, 7(6):833-837.

4. Jeong YI, Kang MK, Sun HS, Kang SS, Kim HW, Moon KS, Lee KJ, Kim $\mathrm{SH}$, Jung S: All-trans-retinoic acid release from core-shell type nanoparticles of poly(epsilon-caprolactone)/poly(ethylene glycol) diblock copolymer. Int J Pharm 2004, 273( I-2):95- 107.

5. Dong AJ, Deng LD, Sun DX, Zhang YT, Jin JZ, Yuan Y]: [Studies on paclitaxel-loaded nanoparticles of amphiphilic block copolymer]. Yao Xue Xue Bao 2004, 39(2): I49-I52.

6. Jeong YI, Nah JW, Lee HC, Kim SH, Cho CS: Adriamycin release from flower-type polymeric micelle based on star-block copolymer composed of poly(gamma-benzyl L-glutamate) as the hydrophobic part and poly(ethylene oxide) as the hydrophilic part. Int J Pharm I999, I 88( I):49-58.

7. Li S, Jiang WQ, Wang AX, Guan ZZ, Pan SR: [Studies on 5-FU/ PEG-PBLG nano-micelles: preparation, characteristics, and drug releasing in vivo]. Ai Zheng 2004, 23(4):38I-385.

8. Zhou J], Liu J, Xu B: Relationship between lactone ring forms of HCPT and their antitumor activities. Acta Pharmacol Sin 200I, 22(9):827-830.

9. Ding XQ, Wang AX, Kong QY, Chen HZ, Chen Y: [Anticancer effect of hydroxycampothecin on oral squamous carcinoma cell line]. Ai Zheng 2002, 2 I (4):388-39I.

10. Kim TE, Park SY, Hsu CH, Dutschman GE, Cheng YC: Synergistic antitumor activity of troxacitabine and camptothecin in selected human cancer cell lines. Mol Pharmacol 2004, 66(2):285-292.

II. Ping YH, Lee HC, Lee JY, Wu PH, Ho LK, Chi CW, Lu MF, Wang JJ: Anticancer effects of low-dose I0-hydroxycamptothecin in human colon cancer. Oncol Rep 2006, I 5(5): I 273-I 279.

12. Li YF, Zhang R: Reversed-phase high-performance liquid chromatography method for the simultaneous quantitation of the lactone and carboxylate forms of the novel natural product anticancer agent I0-hydroxycamptothecin in biological fluids and tissues. I Chromatogr B Biomed Appl 1996, 686(2):257-265.

13. Machida Y, Onishi H, Kurita A, Hata H, Morikawa A: Pharmacokinetics of prolonged-release CPT-I I-loaded microspheres in rats. J Control Release 2000, 66(2-3): 159-175.

14. Onishi H, Machida Y: Antitumor properties of irinotecan-containing nanoparticles prepared using poly(DL-lactic acid) and poly(ethylene glycol)-block-poly(propylene glycol)block-poly(ethylene glycol). Biol Pharm Bull 2003, 26(I ): I |6-I I9.

15. Williams J, Lansdown R, Sweitzer R, Romanowski M, LaBell R, Ramaswami R, Unger E: Nanoparticle drug delivery system for intravenous delivery of topoisomerase inhibitors. I Control Release 2003, 91 (I-2): I67-I72.

16. Zhang ZR, Lu W: [Study on liver targeting and sustained release hydroxycamptothecin polybutylcyanoacrylate nanoparticles]. Yao Xue Xue Bao I997, 32(3):222-227.

17. Zhang $X$, Pan SR, Hu HM, Wu GF, Feng $M$, Zhang $W$, Luo $X$ : Poly(ethylene glycol)-block-polyethylenimine copolymers as carriers for gene delivery: Effects of PEG molecular weight and PEGylation degree. J Biomed Mater Res A 2007.

18. Sanchez A, Tobio M, Gonzalez L, Fabra A, Alonso MJ: Biodegradable micro- and nanoparticles as long-term delivery vehicles for interferon-alpha. Eur J Pharm Sci 2003, I 8(3-4):221-229.

19. Zhang R, Li Y, Cai Q, Liu T, Sun H, Chambless B: Preclinical pharmacology of the natural product anticancer agent I0hydroxycamptothecin, an inhibitor of topoisomerase I. Cancer Chemother Pharmacol 1998, 4 I (4):257-267.

20. Wang AX, Li S, Ding XQ, Chen Y, Chen D: [Antitumor effects of ring-closed and ring-opened hydroxycamptothecin on oral squamous carcinoma cell line Tca8II3]. Ai Zheng 2005, 24(8):970-974. 